\title{
Alterações corneanas pós-tracoma não associadas a entrópio ou triquíase
}

\author{
Corneal findings not related to entropion or trichiasis after trachoma
}

\author{
Alessandra Pinheiro Chaves ${ }^{1}$ \\ José Alvaro Pereira Gomes ${ }^{2}$ \\ Denise de Freitas ${ }^{3}$
}

${ }^{1}$ Pós-graduanda (Doutorado) do Departamento de Oftalmologia da Universidade Federal de São Paulo.

${ }^{2}$ Doutor em oftalmologia e colaborador do Setor de Doenças Externas e Córnea do Departamento de Oftalmologia da Universidade Federal de São Paulo.

${ }^{3}$ Livre docente em Oftalmologia e Chefe do Setor de Doenças Externas e Córnea do Departamento de Oftalmologia da Universidade Federal de São Paulo.

Endereço para correspondência: Alessandra Pinheiro Chaves - R. João da Silva Campos, 1244 - Salvador (BA) CEP 41815-200.

\begin{tabular}{|l|}
\hline RESUMO \\
\hline Objetivo: Descrever alterações corneanas pós-tracoma não associadas \\
a deformidades palpebrais. Métodos: Foi realizada avaliação oftalmo- \\
lógica completa em 7 pacientes do ambulatório de Córnea e Doenças \\
Externas da Escola Paulista de Medicina da Universidade Federal de \\
São Paulo. Para o nosso estudo selecionamos pacientes com diag- \\
nóstico prévio de tracoma sem deformidades palpebrais, história pre- \\
gressa de correção de entrópio ou epilação, mas com opacidades \\
corneanas. Resultados: Dentre os achados oftalmológicos observa- \\
dos neste estudo destacaram-se: opacidade corneana bilateral em \\
$100 \%$ dos casos, afinamento corneano em $85,7 \%$ dos casos, pannus \\
superior bilateral em 71,4\% dos casos e fibrose da conjuntiva tarsal \\
superior bilateral em 85,7\% dos casos. Conclusão: As alterações \\
corneanas pós-tracomatosas podem-se manifestar sob diversas for- \\
mas clínicas, incluindo opacidades, afinamentos, ceratites secundá- \\
rias a infecção, vas-cularização, ectasias, alteração da sensibilidade \\
e xerose. Na ausência de entrópio ou triquíase, estas alterações \\
podem resultar de inflamação tracomatosa, ceratite intersticial ou \\
formas de tratamento utilizadas no passado, no entanto a sua fisio- \\
patologia ainda é controversa.
\end{tabular}

Descritores: Opacidade de córnea; Tracoma; Entrópio

\section{INTRODUÇÃO}

Tracoma é uma ceratoconjuntivite bilateral causada pela Chlamydia trachomatis, caracterizada por folículos na fase aguda e cicatrização conjuntival na fase crônica. Suas manifestações clínicas resultam da combinação de diferentes fatores, incluindo a espécie e a cepa do agente etiológico, o número de recidivas, infecções bacterianas secundárias, resposta do hospedeiro e fatores ambientais ${ }^{(1)}$.

Em 1987, foi adotado pela Organização Mundial de Saúde um esquema de classificação dos diferentes estágios do tracoma, designando-se "TF" (tracoma inflamatório folicular) quando há presença de 5 ou mais folículos $\geq 0,5 \mathrm{~mm}$ na conjuntiva tarsal superior; "TI" (tracoma inflamatório intenso) quando observa-se hiperplasia papilar da conjuntiva tarsal superior causando espessamento conjuntival que dificulta a observação dos vasos tarsais profundos; "TS" (tracoma cicatricial) quando são observadas linhas de fibrose na conjuntiva tarsal; "TT" (triquíase tracomatosa) quando há pelo menos um cílio em triquíase ou evidência de remoção recente e "CO" (opacidade corneana) na presença de cicatrização corneana envolvendo ao menos parcialmente a área pupilar ${ }^{(2)}$. 


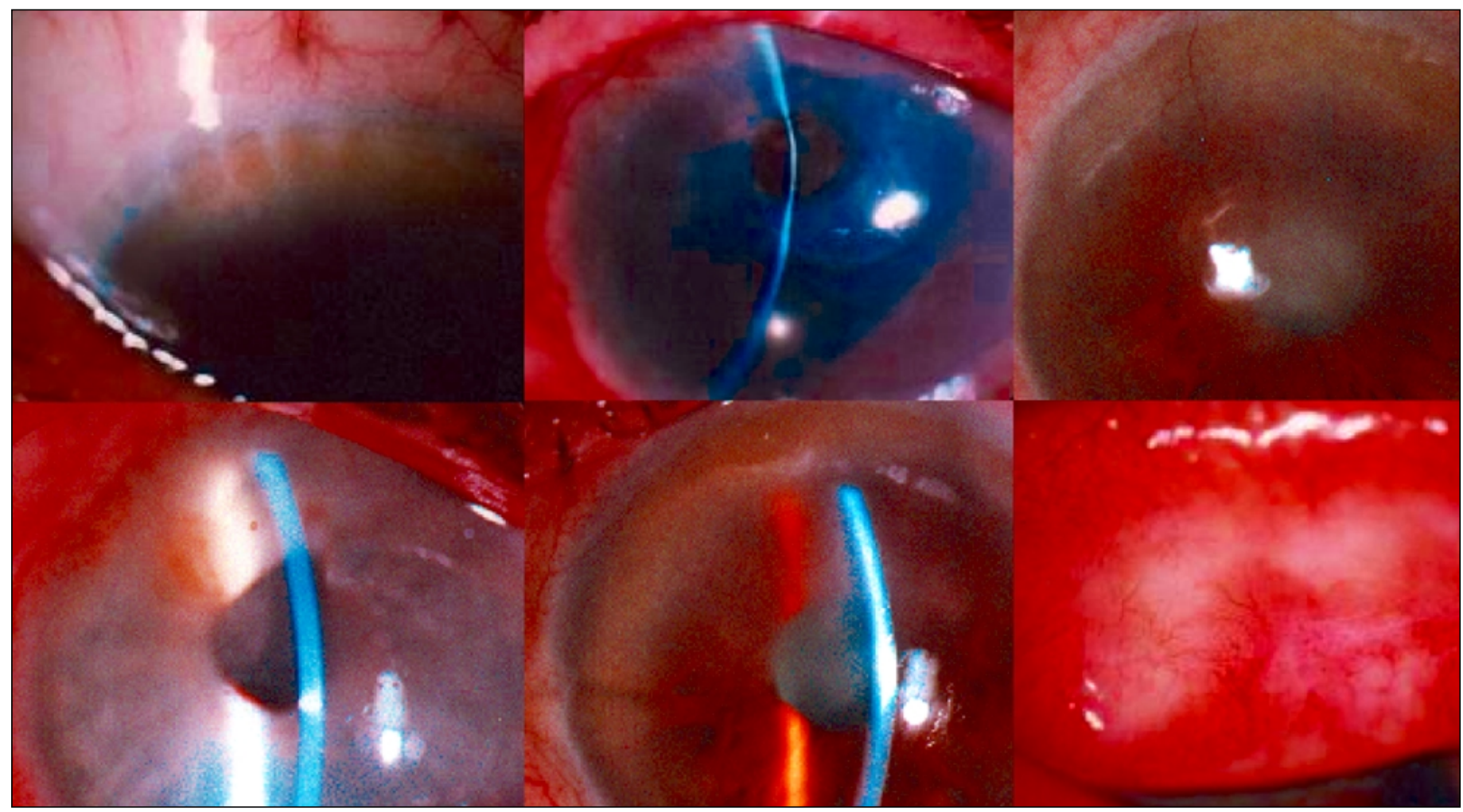

Figura 1 - Superior - Esquerda: Fossetas de Herbert; Centro: Leucoma e afinamento paracentral superior com descemetocele; Direita: Opacidade corneana (leucoma). Inferior - Esquerda e centro: Opacidades corneanas (nébula); Direita: Fibrose da conjuntiva tarsal superior

Embora o diagnóstico do tracoma seja classicamente clínico, vários métodos laboratoriais são testados na tentativa de melhor exploração da população. A investigação laboratorial do tracoma baseia-se na identificação do agente etiológico, no isolamento do agente em cultura celular e na pesquisa de anticorpos clamidianos no sangue e lágrima ${ }^{(3)}$.

As alterações cicatriciais induzidas pelo tracoma podem estar associadas a complicações oculares graves e inclusive levar à cegueira. Dentre essas alterações, destacam-se deformidades palpebrais e triquíase, que podem causar abrasão, úlceras e fibrose corneana, com conseqüente comprometimento visual ${ }^{(3)}$. A inflamação conjuntival pode ainda causar destruição das células caliciformes e obstrução das glândulas lacrimais e tarsais, induzindo olho seco e queratinização da córnea com perda visual resultante. Alguns pacientes, entretanto, apresentam alterações corneanas importantes, que não são compatíveis com os achados cicatriciais das pálpebras, conjuntiva ou olho seco. Existem poucos relatos na literatura que mencionam tais alterações corneanas secundárias ao tracoma. O objetivo deste trabalho é mostrar alterações corneanas pós-tracoma na ausência de entrópio ou triquíase e discutir a sua fisiopatologia.

\section{MÉTODOS}

Os pacientes deste estudo foram selecionados no ambulatório de Córnea e Doenças Externas da Escola Paulista de
Medicina da Universidade Federal de São Paulo. Para o nosso estudo selecionamos pacientes com diagnóstico clínico prévio de tracoma sem deformidades palpebrais, história pregressa de correção de entrópio ou epilação, mas com opacidades e afinamentos corneanos. Estudos laboratoriais não foram realizados, pois nenhum dos pacientes apresentava tracoma inflamatório ou folicular. A avaliação oftalmológica completa incluiu cuidadoso exame biomocroscópico em ambos os olhos dos 7 pacientes.

\section{RESULTADOS}

Foram observadas as seguintes alterações: opacidade corneana bilateral em 100\% dos casos (nébula em 6 olhos e leucoma em 8 olhos); afinamento corneano em $85,7 \%$ dos casos (central em 5 olhos, paracentral em 4 olhos e difuso em 1 olho, sendo bilateral em 4 casos e unilateral em 2 casos); pannus superior bilateral em 71,4\% dos casos; fibrose da conjuntiva tarsal superior bilateral em 85,7\% dos casos (Figura 1). Outras alterações observadas foram catarata e achados fundoscópicos (Quadros 1 e 2). Tais alterações isoladas ou associadas justificam a BAV apresentada pelos pacientes no presente estudo, no entanto vale ressaltar a forte associação entre o tracoma e as alterações corneanas observadas na ausência de deformidades palpebrais, achado pouco relatado na literatura científica. 


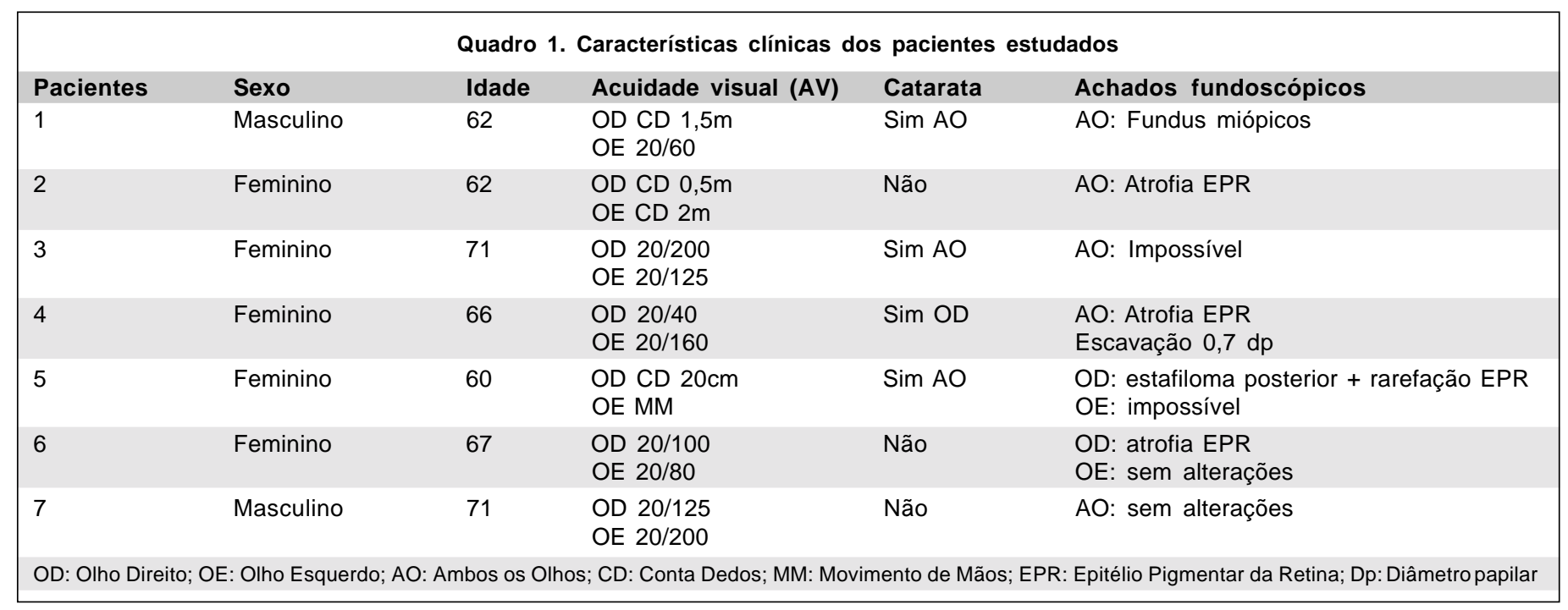

\begin{tabular}{|c|c|c|c|c|c|c|c|}
\hline \multicolumn{8}{|c|}{ Quadro 2. Principais alterações oculares observadas } \\
\hline $\begin{array}{l}\text { Olhos e } \\
\text { pacientes }\end{array}$ & $\begin{array}{l}\text { Fossetas de } \\
\text { Herbert }\end{array}$ & Pannus & $\begin{array}{l}\text { Alterações } \\
\text { conjuntivais }\end{array}$ & $\begin{array}{l}\text { Alterações } \\
\text { palpebrais }\end{array}$ & $\begin{array}{l}\text { Opacidade } \\
\text { corneana }\end{array}$ & $\begin{array}{l}\text { Afinamento } \\
\text { corneano }\end{array}$ & $\begin{array}{l}\text { Vascularização } \\
\text { corneana }\end{array}$ \\
\hline $1 \mathrm{OD}$ & - & Superior & $\begin{array}{l}\text { Fibrose da conjuntiva } \\
\text { tarsal superior }\end{array}$ & - & Leucoma central & Central & Superior \\
\hline $1 \mathrm{OE}$ & - & Superior & $\begin{array}{l}\text { Fibrose da conjuntiva } \\
\text { tarsal superior }\end{array}$ & - & Leucoma central & Central & Superior \\
\hline $2 \mathrm{OD}$ & - & - & $\begin{array}{l}\text { Fibrose da conjuntiva } \\
\text { tarsal superior }\end{array}$ & - & $\begin{array}{l}\text { Opacidade nas } \\
\text { córneas doadora } \\
\text { e receptora }+ \\
\text { edema } 2+/ 4+\end{array}$ & - & - \\
\hline $2 \mathrm{OE}$ & - & - & $\begin{array}{l}\text { Fibrose da conjuntiva } \\
\text { tarsal superior }\end{array}$ & - & Difusa & $\begin{array}{l}\text { Difuso, mais } \\
\text { acentuado na } \\
\text { região central }\end{array}$ & - \\
\hline $3 \mathrm{OD}$ & - & Superior & $\begin{array}{l}\text { Fibrose da conjuntiva } \\
\text { tarsal superior }\end{array}$ & - & Leucoma central & Central & Superior \\
\hline $3 \mathrm{OE}$ & - & Superior & $\begin{array}{l}\text { Fibrose da conjuntiva } \\
\text { tarsal superior }\end{array}$ & - & Leucoma central & Central & Superior \\
\hline $4 \mathrm{OD}$ & + & Superior & $\begin{array}{l}\text { Fibrose da conjuntiva } \\
\text { tarsal superior }\end{array}$ & Blefarite & Nébula central & Superior & Superior \\
\hline $4 \mathrm{OE}$ & - & Superior & $\begin{array}{l}\text { Fibrose da conjuntiva } \\
\text { tarsal superior }\end{array}$ & Blefarite & Leucoma difuso & $\begin{array}{l}\text { Paracentral } \\
\text { superior, com } \\
\text { descemetocele }\end{array}$ & Superior \\
\hline $5 \mathrm{OD}$ & - & Superior & Simbléfaro & - & $\begin{array}{l}\text { Nébula } \\
\text { paracentral }\end{array}$ & Paracentral & $\begin{array}{l}\text { Neovasos } \\
\text { superficiais } \\
\text { na periferia }\end{array}$ \\
\hline $5 \mathrm{OE}$ & - & Superior & Simbléfaro & - & $\begin{array}{l}\text { Leucoma } \\
\text { paracentral }\end{array}$ & Paracentral & $\begin{array}{l}\text { Neovasos } \\
\text { superficiais } \\
\text { na periferia }\end{array}$ \\
\hline $6 \mathrm{OD}$ & + & - & $\begin{array}{l}\text { Fibrose da conjuntiva } \\
\text { tarsal superior }\end{array}$ & Meibomite & Nébula difusa & - & $\begin{array}{l}\text { Neovasos } \\
\text { superficiais } \\
\text { na periferia }\end{array}$ \\
\hline $6 \mathrm{OE}$ & + & - & $\begin{array}{l}\text { Fibrose da conjuntiva } \\
\text { tarsal superior }\end{array}$ & Meibomite & Nébula difusa & - & $\begin{array}{l}\text { Neovasos } \\
\text { superficiais } \\
\text { na periferia }\end{array}$ \\
\hline $7 \mathrm{OD}$ & - & Superior & $\begin{array}{l}\text { Fibrose da conjuntiva } \\
\text { tarsal superior }\end{array}$ & - & $\begin{array}{l}\text { Periférica (na } \\
\text { córnea receptora) }\end{array}$ & - & Superior \\
\hline $7 \mathrm{OE}$ & - & Superior & $\begin{array}{l}\text { Fibrose da conjuntiva } \\
\text { tarsal superior }\end{array}$ & - & Nébula difusa & Central & Superior \\
\hline
\end{tabular}




\section{DISCUSSÃO}

Classicamente, o tracoma causa fibrose conjuntival, que pode resultar em olho seco, triquíase e entrópio. Outro achado comum consiste em opacidade corneana difusa, que traduz o estágio final dos infiltrados corneanos ${ }^{(4)}$. A cegueira secundária ao tracoma ocorre geralmente devido a leucomas centrais, associados a cicatrizes conjuntivais e triquíase. Em alguns casos a inflamação tracomatosa pode levar a uma ceratite supurativa aumentando as chances de infecção secundária ${ }^{(5)}$.

Diallo et al ${ }^{(6)}$ classificaram as alterações corneanas póstracomatosas em 5 tipos: modificação da curvatura e da espessura, modificação da transparência, vascularização corneana residual, alterações da sensibilidade corneana e xerose corneana. Bhatia et al. ${ }^{(7)}$ relataram alterações na curvatura corneana em 141 casos, relacionando-as ao estágio evolutivo da doença, sendo maior o grau de ectasia nos casos mais avançados. Quanto à localização, as opacidades corneanas, podem ser classificadas em periféricas, centrais e difusas ${ }^{(8)}$. Há também relato de ceratite intersticial e degeneração lipídica associadas a glaucoma ${ }^{(9-10)}$. Hardten et al. ${ }^{(11)}$ relataram 2 casos de ceratite ponteada persistente após ceratoconjuntivite por Chlamydia trachomatis, sendo descritas lesões bilaterais acinzentadas e recorrentes em vários níveis no epitélio corneano, bem como edema estromal anterior em um dos casos.

Nos anos 30, várias drogas e métodos eram utilizados no tratamento do tracoma, tais como: cauterização pelo sulfato de cobre e nitrato de prata, solução de sulfato de cobre puro, solução de nitrato de mercúrio, solução de azul de metileno, solução de sulfato de zinco, solução de sulfato de iodo, injeção subconjuntival de cobre coloidal, jequiriti, diatermia, irradiação solar por meio de uma lente e destruição mecânica das granulações ${ }^{(12)}$. Atualmente, drogas como tetraciclina, eritromicina, azitromicina e ciprofloxacina têm sido utilizadas no tratamento do tracoma, mas sua erradicação depende basicamente da melhoria das condições de higiene e saúde ${ }^{(13)}$.

Morschbacher et al. (1996) ${ }^{(14)}$ realizou um estudo sobre a prevalência de tracoma entre os habitantes do Parque Indígena do Xingu. De acordo com os achados de lesões tracomatosas evidenciadas em pelo menos um dos olhos, foi procedida a classificação segundo o esquema adotado pela OMS. Foram examinados 1272 índios, sendo a prevalência total de tracoma na população examinada de $33,8 \%$, variando entre $18,0 \%$ e $75,2 \%$ entre as diferentes tribos. Não foram encontrados casos de triquíase, entrópio, opacificação de córnea ou baixa de acuidade visual provocada por tracoma. O tracoma não associado a complicações cicatriciais importantes, é endêmico entre os índios do sudoeste norte-americano; apesar da alta prevalência, não há seqüelas importantes. Este mesmo perfil da doença foi encontrado entre os índios examinados no Parque Indígena do Xingu, o que mostra um paralelismo entre o comportamento do tracoma das duas populações. Em áreas endêmicas, o tracoma exibe um mesmo padrão em relação à idade. A fase ativa da doença tem um pico de prevalência entre as crianças na faixa etária de 0 a 10 anos; enquanto a prevalência da forma ativa vai decrescendo, a prevalência de tracoma cicatricial vai aumentando com a idade, sendo que os achados cicatriciais são encontrados na população adulta ${ }^{(14)}$.

A cicatrização conjuntival no tracoma resulta da intensidade e duração do processo inflamatório. A doença inflamatória, mesmo de baixa intensidade, persistente por um longo tempo, leva a um acúmulo de cicatrizes conjuntivais, produzidas desde a infância, que através de suas retrações podem ocasionar triquíase e entrópio ${ }^{(1)}$. Em nosso estudo, relatamos 7 casos com alterações conjuntivais pós-tracoma associadas a BAV e opacidade corneana, sem deformidades palpebrais associadas. Tais alterações podem promover lesões corneanas acumulativas anos após o aparecimento das cicatrizes que colaboram para a gravidade do tracoma determinando se a moléstia tem características de doença que cega ou não cega $^{(1)}$. Dentre as lesões corneanas observadas, destacamos a presença de afinamentos corneanos, os quais muitas vezes apresentam aspecto de ceratocone invertido devido à sua localização superior; destacamos ainda a presença de opacificações corneanas, muitas vezes responsáveis pela diminuição da acuidade visual dos pacientes. Muitas formas de tratamento utilizadas no passado podem ter sido responsáveis por queimaduras químicas oculares. Isto talvez justifique alguns casos de alterações corneanas associadas a afinamentos, já que muitos desses pacientes são idosos e foram tratados há mais de 20 anos. No entanto, nenhum dos nossos pacientes soube relatar qual o tipo de tratamento a que foi submetido. Outra possibilidade é que tais lesões sejam decorrentes de ceratite por Chlamydia associada ou não a infecção secundária.

Em resumo, relatamos casos de sem deformidades palpebrais e que apresentam alterações corneanas importantes com comprometimento da AV. As alterações corneanas pós-tracomatosas podem se manifestar sob diversas formas clínicas, incluindo opacidades, afinamentos, ceratites secundárias a infecção, vascularização, ectasias, alteração da sensibilidade e xerose. Na ausência de entrópio ou triquíase estas alterações podem resultar de inflamação tracomatosa, ceratite intersticial ou formas de tratamento utilizadas no passado, entretanto, mais estudos são necessários para elucidar a etiopatogenia destas alterações.

\section{ABSTRACT}

Purpose: To describe corneal findings not related to eyelid involvement after trachoma. Methods: Complete ocular evaluation was performed in 7 patients from the Cornea and External Diseases Clinics of the Escola Paulista de Medicina Universidade Federal de São Paulo. The selected patients had a previous diagnosis of trachoma with no eyelid deformities, previous entropion repair or trichiasis, but presented corneal opacities. Results: The most common findings were bilateral corneal opacities in $100 \%$ of the cases, corneal thinning in 
$85.7 \%$ of the cases, bilateral superior pannus in $71.4 \%$ of the cases and bilateral superior tarsal conjunctiva fibrosis in $85.7 \%$ of the cases. Conclusion: Corneal findings after trachoma may be present different clinical aspects, including opacities, thinning, infectious keratitis, vascularization, ectasy, sensibility decrease and xerosis. If entropion and trichiasis are absent, these findings may result from trachomatous inflamation, interstitial keratitis or forms of treatment performed in the past. Nevertheless their pathophysiology is still controversial.

Keywords: Corneal opacity; Trachoma; Entropion

\section{REFERÊNCIAS}

1. Scarpi MJ, Gentil R. Sinais e sintomas do tracoma em povoado do Estado da Bahia-Brasil. Arq Bras Oftalmol 1990;53:276-8.

2. Thylefors B, Dawson CR, Jones BR, West SK, Taylor HR. A simple system for assessment of trachoma and its complications. Bull World Health Organ $1987 ; 65: 477-83$.
3. Darougar S, Jones BR. Trachoma. Br Med Bull 1983;39:117-22.

4. Goldstein M, Lowenstein A, Rosner M, Lipshitz I, Lazar M. Phototherapeutic keratectomy in the treatment of corneal scarring from trachoma. J Refract Corneal Surg 1994;10(2 suppl):S290-2.

5. Thylefors B. Contrôle Global du Trachome. Passé, présent et futur. Rev Int Trach Pathol Ocul Trop Subtrop Santé Publique 1995;72:17-80.

6. Diallo JS, Cornand GA. Post trachomatous degeneration. Rev Int Trach Pathol Ocul Trop Subtrop 1978;55:5-186.

7. Bhatia RP, Awasthy P. Corneal curvature and trachoma. Ann Ophthalmol 1983;15:372-4.

8. Hosni FA. Clinical aspects of corneal trachoma. Br J Ophtalmol 1978;62:159-62.

9. Soliman AM. Trachomatous interstitial keratitis. Rev Int Trach 1972;49:51-5.

10. Soliman AM. Fatty degeneration in trachomatous cornea. Rev Int Trach 1972;49:56-61.

11. Hardten DR, Doughman DJ, Holland EJ, Gothard TW. Persistent superficial punctate keratitis after resolution of chlamydial follicular conjuntivitis. Cornea 1992;11:360-3.

12. Scarpi MJ. História do tracoma no Brasil. Arq Bras Oftalmol 1991;54:202-5.

13. Adan CBD, Scarpi MJ, Guidugli T. Eficácia da ciprofloxacina e da tetraciclina no tratamento do tracoma: Estudo clínico e microbiológico. Arq Bras Oftalmol 1996;59:592-600.

14. Morschbacher R, Scarpi M. Prevalência de tracoma no parque indígena do Xingu. Arq Bras Oftalmol 1996;59:83-7.

15. Scarpi M, Gentil R. Sinais e sintomas do tracoma em povoado do Estado da Bahia-Brasil. Arq Bras Oftalmol 1990;53:276-8. 